\title{
Superoleophobic Surfaces on Stainless Steel Substrates Obtained by Chemical Bath Deposition
}

\author{
Liu Huang ${ }^{l}$, Jinlong Song ${ }^{1}$, Yao Lu ${ }^{2}$, Faze Chen ${ }^{1}$, Xin Liu ${ }^{1}$, Zhuji Jin ${ }^{1}$, Danyang Zhao ${ }^{1}$, Claire J. Carmalt ${ }^{2}$, \\ Ivan P. Parkin ${ }^{2}$ \\ ${ }^{1}$ Key Laboratory for Precision and Non-traditional Machining Technology of the Ministry of Education, Dalian University of \\ Technology, Dalian 116024, People's Republic of China. \\ ${ }^{2}$ Materials Chemistry Research Centre, Department of Chemistry, University College London, 20 Gordon Street, \\ London WCIH OAJ, UK \\ E-mail:songjinlong@dlut.edu.cn (J.L.Song)
}

\begin{abstract}
Superhydrophobic surfaces have shown great potential in domestic and industrial applications. However, these surfaces lose their superhydrophobic functions once being contaminated by oily liquids. In this study, a simple chemical bath deposition method is reported to fabricate superoleophobic surfaces on steel substrates that repel both water and oil. The synthesis of superoleophobic surfaces involves the fabrication of the micro/nanometerscale origami-ball-like structures on steel substrates, followed by the modification of low surface energy material. The fabricated surfaces have glycerol, peanut oil and hexadecane contact angles larger than $150^{\circ}$ and roll-off angles smaller than $10^{\circ}$. This method is highly efficient because it takes only 5 mins to create the surface re-entrant structures that are required by superoleophobicity. The prepared surfaces showed remarkable durability and retained superoleophobicity even after exposure to high and low temperatures ( -30 and $\left.100^{\circ} \mathrm{C}\right)$, and UV irradiation. This work will enrich the processing methods of the superoleophobic surfaces on stainless steel substrates.
\end{abstract}

1. Introduction: Superhydrophobic surfaces with water contact angle (CA) larger than $150^{\circ}$ and roll-off angle (RA) smaller than $10^{\circ}$ have attracted researchers' extensive attention due to their diverse applications in self-cleaning,[1-3] anti-icing,[4-10] oil/water separation,[11-15] corrosion resistance, $[16,17]$ and liquid directional transportation.[18-20] Microscopic rough structures and low surface energy are necessary for the fabrication of superhydrophobic surfaces.[21, 22] To date, researchers have developed many ways to fabricate superhydrophobic surfaces on various substrates including metal alloys,[23-25] cotton,[26] glass,[27, 28] wood,[29] and fiber.[30, 31] However, superhydrophobic surfaces are usually contanminated in oily circumstances and thus lose their functionality, which greatly hinder their applications. Therefore, the fabrication of superoleophobic surfaces with oil CA larger than $150^{\circ}$ and RA smaller than $10^{\circ}$ is urgently needed. However, the synthesis of superoleophobic surfaces requires the creation of surface re-entrant structures, $[32,33]$ which is more difficult to achieve compared with the micro/nano surface morphology of superhydrophobic surfaces. The existing research results about superoleophobic surfaces are far less than superhydrophobic surfaces. According to Web of Science, the number of the published research papers about superhydrophobic surfaces and their applications are increasing exponentially year by year, but the paper number of superoleophobic surfaces are growing much slowly (Fig. 1). For example, 1578 articles of superhydrophobic surfaces is retrieved in Web of Science in 2015, while there are only 146 papers about superoleophobic materials. Tuteja et al.[32, 34] revealed that the re-entrant structures are crucial to obtain superoleophobicity. Since then, researchers developed some methods to fabricate superoleophobic surfaces: Ahuja et al.[35] used ion etching to construct silicon nanonail structures with re-entrant effect and obtained superoleophobic toward a variety of oils such as methanol $\left(\gamma_{\mathrm{LV}}=22.1 \mathrm{mN} / \mathrm{m}\right)$, 1-propanol $\left(\gamma_{\mathrm{LV}}=23.7 \mathrm{mN} / \mathrm{m}\right)$ and 1decanol $\left(\gamma_{\mathrm{LV}}=28.5 \mathrm{mN} / \mathrm{m}\right)$ after being coated by an additional conformal smooth fluorocarbon layer; Song et al.[36] used electrochemical etching and $\left[\mathrm{Ag}\left(\mathrm{NH}_{3}\right)_{2}\right]^{+}$solution immersion method to construct re-entrant micro/nanometer-scale rough structures on aluminum substrate, and the obtained surfaces became superoleophobic to peanut oil $\left(\gamma_{\mathrm{LV}}=34.5 \mathrm{mN} / \mathrm{m}\right)$ with $\mathrm{CA}$ of $160 \pm 2^{\circ}$ and RA of $8^{\circ}$ after perfluorooctanoic acid (PFOA) modification; Chen et al.[37] constructed $\mathrm{Cu}$ powder with re-entrant textures composed of blocky, dendritic and coralline-shaped structures by chemical deposition and the powder showed excellent superoleophobicity after modification with $0.015 \mathrm{~mol} / \mathrm{L}$ aqueous PFOA solution.

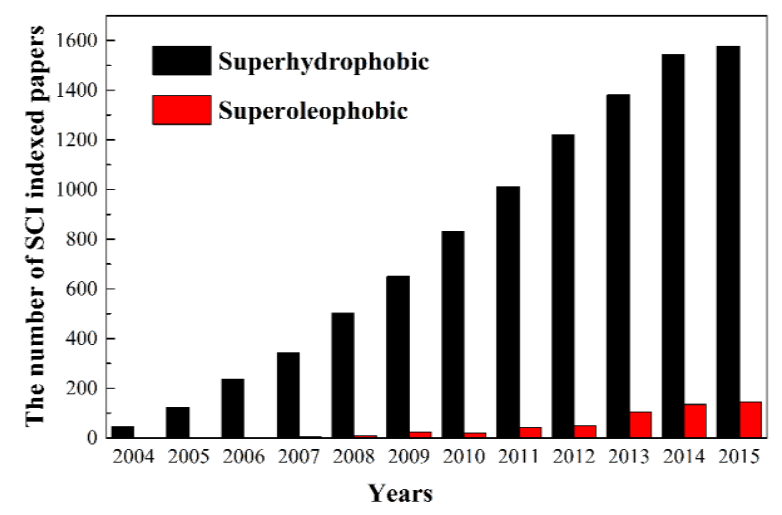

Fig. 1 The statistics of the paper indexed in the ISI web of science by the topic of superhydrophobic and superoleophobic

Steel is one of the most widely used materials in our daily life, construction and manufacture industry due to their low price and high reliability. The fabrication of superoleophobic surfaces on steel substrates has great significance for both fundamental research and industrial applications. However, only a few methods were reported to fabricate superoleophobic surfaces on steel substrates. For example, Yuan et al.[38] immersed cleaned cast iron substrate into 36\% acetic acid solution for $2 \mathrm{~h}$ and $15 \% \mathrm{H}_{2} \mathrm{O}_{2}$ solution for $3 \mathrm{~h}$ in turn to obtain microflower-like microstructures, followed by the modification with ethanol solution of perfluorocarboxylic acid, the surface became super-repellent toward water $\left(\gamma_{\mathrm{LV}}=72.1 \mathrm{mN} / \mathrm{m}\right)$ and rapeseed oil $\left(\gamma_{\mathrm{LV}}=35.7 \mathrm{mN} / \mathrm{m}\right)$. Valipour et al.[39] fabricated multi-scale roughness structures composed of spherical silica particles on stainless steel by sandblasting and then silica particles coating. The silica particles of upper layer obtained superoleophobicity, and the 
CAs of ethylene glycol $\left(\gamma_{\mathrm{LV}}=63.6 \mathrm{mN} / \mathrm{m}\right)$ and fuel oil $\left(\gamma_{\mathrm{LV}}=35.3 \mathrm{mN} / \mathrm{m}\right)$ were respectively $157^{\circ}$ and $116^{\circ}$ after being modified with fluoropolymer. However, the above mentioned methods are either time-consuming or performed poorly in contact with oils. Therefore, it is important to develop a facile, efficient and adaptable technique to fabricate stable superoleophobic surfaces on steel substrates that repel a range of liquids such as water and oils.

In this work, we report a simple method to prepare superoleophobic stainless steel surfaces that repelled glycerol, peanut oil and hexadecane effectively. The superoleophobic surfaces were achieved through the creation of re-entrant micro/nanometer-scale origami-balllike rough structures using chemical bath deposition for only 5 mins, followed by the low surface energy modification using PFOA. The prepared surfaces showed remarkable durability and retained superoleophobicity even after exposure to high and low temperatures $\left(-30\right.$ and $\left.100^{\circ} \mathrm{C}\right)$, and UV irradiation. This facile, efficient and low cost method has great potential for industrial applications even in harsh environments.

\section{Experimental}

2.1 Materials: 304 stainless steel plates $(20 \mathrm{~mm} \times 20 \mathrm{~mm} \times 2 \mathrm{~mm})$ were purchased from Shenzhen Metal Manufacturer (China). Hydrochloric acid, ammonia, potassium persulfate $\left(\mathrm{K}_{2} \mathrm{~S}_{2} \mathrm{O}_{8}\right)$, and nickel sulfate $\left(\mathrm{NiSO}_{4} \cdot 6 \mathrm{H}_{2} \mathrm{O}\right)$ were purchased from Tianjin Kermel Chemical Reagent Co. (China). Perfluorooctanoic acid (PFOA, $\left.\mathrm{CF}_{3}\left(\mathrm{CF}_{2}\right)_{6} \mathrm{COOH}\right)$ was purchased from Sigma-Aldrich (USA). Hexadecane $\left(\mathrm{C}_{16} \mathrm{H}_{34}\right.$, purity $\left.>98 \%\right)$ was purchased from Aladdin Industrial Co. (USA). Peanut oil was purchased from Shandong Luhua Co. (China). The rest of the chemical reagents used in this experiment were of analytical reagent (AR) and purchased from Tianjin Kermel Chemical Reagent Co. (China). In the experiment, water $\left(\gamma_{\mathrm{LV}}=72.1 \mathrm{mN} / \mathrm{m}\right)$, glycerol $\left(\gamma_{\mathrm{LV}}=63.6 \mathrm{mN} / \mathrm{m}\right)$ and hexadecane $\left(\gamma_{\mathrm{LV}}=27.5 \mathrm{mN} / \mathrm{m}\right)$ were respectively dyed blue, green and red to aid visualization while peanut oil $\left(\gamma_{\mathrm{LV}}=34.5 \mathrm{mN} / \mathrm{m}\right)$ kept its original color, and this almost did not change the surface tension of the droplets.

2.2 Fabrication of superoleophobic surfaces: Prior to the chemical deposition, stainless steel plates were polished mechanically using 800\# and 1500\# metallographic abrasive paper to remove the impurities and the oxide layer on the surfaces. The steel plates were then ultrasonically cleaned in detergent and deionized water in sequence. After that, the steel plates were immersed in hydrochloric acid (about 37\%) for $30 \mathrm{~min}$ for increasing the contact area between the deposition substances and substrate. And plates were then ultrasonically washed by deionized water and dried. Sequentially, the steel plates were placed in the Chemical Bath Deposition (CBD) solution which were composed of $1.0 \mathrm{~mol} / \mathrm{L} \mathrm{NiSO}_{4} \cdot 6 \mathrm{H}_{2} \mathrm{O}, 0.25 \mathrm{~mol} / \mathrm{L}$ $\mathrm{K}_{2} \mathrm{~S}_{2} \mathrm{O}_{8}$, and $25 \%$ ammonia aqueous solutions in a mass ratio of 5:4:1 for $2 \sim 30 \mathrm{~min}$ at room temperature. Finally, the samples were immersed in a $0.015 \mathrm{~mol} / \mathrm{L}$ aqueous PFOA solution for $30 \mathrm{~min}$ to reduce the surface energy.

2.3 Characterization: The surface morphologies of the samples were characterized using a scanning electron microscope (SEM, JSM6360LV, Japan). The surface chemical compositions of the samples were investigated using energy dispersive X-ray spectroscope (EDS, INCA Energy, Oxford Instruments), senior Fourier transform infrared spectrophotometer (FTIR, Thermo Fisher 6700, America) and X-ray photoelectron spectroscopy (XPS, Thermo ESCALAB 250Xi, USA. $\mathrm{Al}-\mathrm{K} \alpha$ radiation and $\mathrm{C} 1 \mathrm{~s}$ peak $(284.6 \mathrm{eV})$ reference). The crystal structures of the samples were examined by an X-ray diffraction meter system (XRD-6000, Japan). The digital images of bouncing behaviors were recorded by a high speed camera (Hot Shot 512 SC camera equipped with a Nikon $105 \mathrm{~mm}$ f/2.8G lens) from NAC Image Technology Inc (USA). The contact angle (CA) and roll-off angle (RA) of the samples were measured using an optical contact angle meter (DSA100, Krüss, Germany) at room temperature by dropping the given droplets of $5 \mu \mathrm{L}$ on samples, and the average of five measurements at different positions was used as the CA value. Similarly, the RA was obtained by the average level of surface inclining angles upon droplets were rolling off.

2.4 High/low temperature storage and UV irradiation tests: High/low temperature storage UV irradiation were conducted to test the durability of the obtained superoleophobic surface. To estimate the high/low temperature stability, CAs and RAs of the obtained superoleophobic surface after storage in different temperatures (-30 to $100^{\circ} \mathrm{C}$ ) for $1 \mathrm{~h}$. Similarly, CAs and RAs were measured after UV irradiation test $(365 \mathrm{~nm}, 32 \mathrm{~W}$ ) for $16 \mathrm{~h}$.

\section{Results and discussion}

3.1 Effect of surface morphology on oleophobicity: Chemical deposition and PFOA modification processes are very important to obtain superoleophobicity. Fig. 2 shows the digital images of water, glycerol, peanut oil and hexadecane on the different stainless steel surfaces, the insets are the images of the liquids on those surfaces. Fig. 2(a) shows that the original stainless steel surface was lyophilic to water and glycerol, and superlyophilic to peanut oil and hexadecane. After chemical deposition for $15 \mathrm{~min}$, the stainless steel surface showed superlyophilic towards the four kinds of liquids, as shown in Fig. 2(b). Especially, when droplets of water and hexadecane were dropped onto the aforementioned surface, they both spread very quickly, showing contact angles of approximately $0^{\circ}$. Fig. 2(c) shows that the samples became superoleophobic after being further modified by PFOA as the four types of liquids remained like spheres and could roll off from the surface easily. The CAs of water, glycerol, peanut oil and hexadecane were $161.0^{\circ}, 164.2^{\circ}, 157.5^{\circ}$ and $155.2^{\circ}$, respectively.
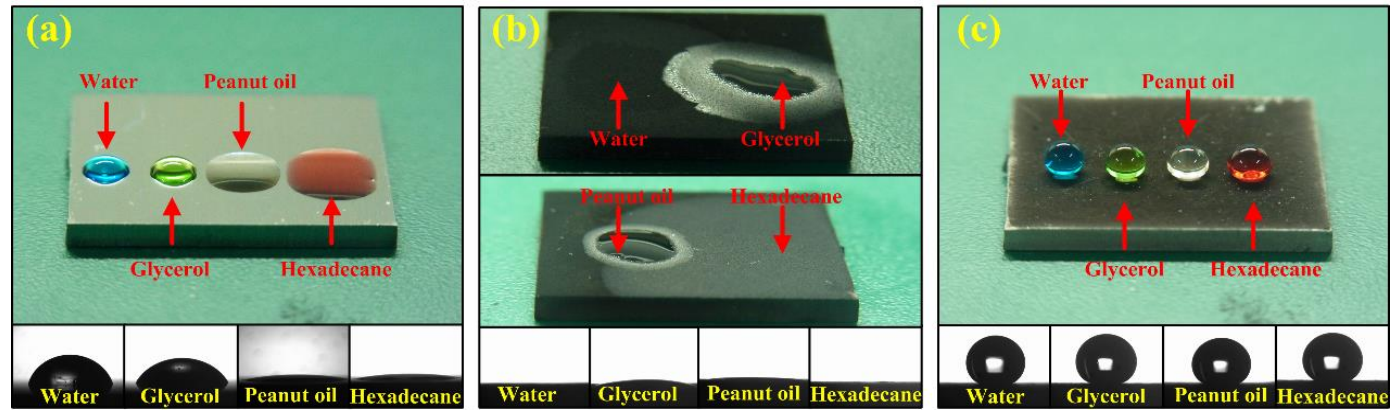

Fig. 2 The digital images of water, glycerol, peanut oil and hexadecane on (a) original stainless steel, (b) 15 min chemically deposited stainless steel surface and (c) 15 min chemically deposited and PFOA-modified stainless steel surface. The insets are the images of the liquids on those surfaces. 
Fig. 3 shows the SEM images, EDS spectra, and XRD patterns of the stainless steel surfaces under different processing conditions. The original stainless steel surface was smooth and only contained a few

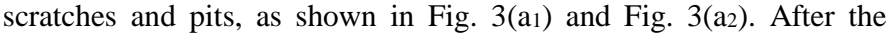
chemical deposition, the hierarchical rough structures composed of many origami-ball-like structures with diameters of 1 to $2 \mu \mathrm{m}$ appeared on the stainless steel substrates (Fig. 3(b $\left.b_{1}\right)$ ). In the sight of larger magnification (Fig. 3(b $\left.b_{2}\right)$ ), origami-ball-like structures were rather rough, and there were many nanoscale porous textures on the spheres. Fig. 3( $\left.c_{1}\right)$ and $3\left(c_{2}\right)$ shows the SEM images of the stainless steel surfaces after chemical deposition for $15 \mathrm{~min}$ and PFOA modification. Comparing the morphologies of the deposited layer before and after fluorination, we could see that the porous textures on the origami ball transformed into thin flake and flocculent structures after PFOA treatment, which demonstrated that chemical reactions might occur in the process of the immersion in aqueous PFOA solution.
After chemical deposition, the peaks of elements $\mathrm{Ni}$ and $\mathrm{O}$ became stronger and new element $\mathrm{S}$ was also detected in the EDS spectra (Fig. $3\left(\mathrm{a}_{3}\right)$ and $\left.3\left(\mathrm{~b}_{3}\right)\right)$. From XRD patterns, new diffraction peaks belong to $3 \mathrm{Ni}(\mathrm{OH})_{2} \cdot 2 \mathrm{H}_{2} \mathrm{O}$ (JCPDS 22-0444), $\beta-\mathrm{Ni}(\mathrm{OH})_{2}$ (JCPDS 14-0117) and $\gamma-\mathrm{NiOOH}$ (JCPDS 06-0075) were detected (Fig. 3(a4) and 3(b4)). Thus, the main compositions of the origami-ball-like structures obtained by chemical deposition were $3 \mathrm{Ni}(\mathrm{OH})_{2} \cdot 2 \mathrm{H}_{2} \mathrm{O}, \mathrm{Ni}(\mathrm{OH})_{2}$ and $\mathrm{NiOOH}$. The detected $\mathrm{S}$ element should be from the attached anion $\mathrm{S}_{2} \mathrm{O}_{8}{ }^{2-}$ and $\mathrm{SO}_{4}{ }^{2-}$ from the CBD solutions.[40] The main chemical reaction equations were shown as follows:

$\left[\mathrm{Ni}(\mathrm{OH})_{6-\mathrm{x}}\left(\mathrm{NH}_{3}\right)_{\mathrm{x}}\right]^{2+}+2 \mathrm{OH}^{-} \rightarrow \mathrm{Ni}(\mathrm{OH})_{2}+(6-\mathrm{x}) \mathrm{H}_{2} \mathrm{O}+\mathrm{xNH}_{3}$ $3\left[\mathrm{Ni}(\mathrm{OH})_{6-\mathrm{x}}\left(\mathrm{NH}_{3}\right)_{\mathrm{x}}\right]^{2+}+6 \mathrm{OH}^{-} \rightarrow$

$3 \mathrm{Ni}(\mathrm{OH})_{2} \cdot 2 \mathrm{H}_{2} \mathrm{O}+(16-3 \mathrm{x}) \mathrm{H}_{2} \mathrm{O}+3 \mathrm{xNH}_{3}(2)$

$2 \mathrm{Ni}(\mathrm{OH})_{2}+\mathrm{S}_{2} \mathrm{O}_{8}{ }^{2-} \rightarrow 2 \mathrm{NiOOH}+2 \mathrm{SO}_{4}{ }^{2-}+2 \mathrm{H}^{+}$
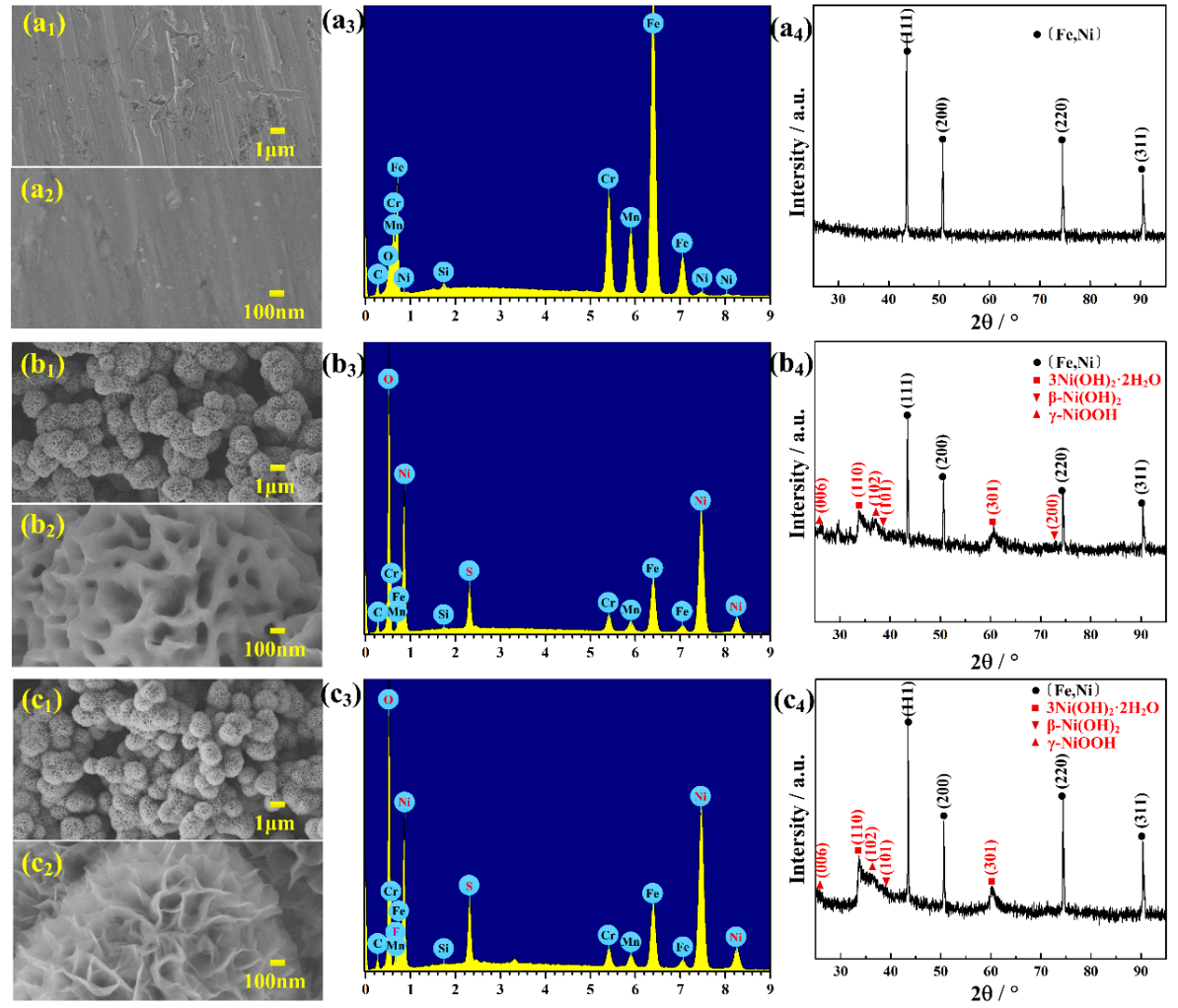

Fig. 3 SEM images, EDS spectra, and XRD patterns of the stainless steel surfaces under different processing conditions: (a) original stainless steel; (b) 15 min chemically deposited stainless steel surface; (c) 15 min chemically deposited and PFOA-modified stainless steel surface.

After PFOA modification, $\mathrm{F}$ appeared on the EDS spectrum (as shown in Fig. 3( $\left.c_{3}\right)$ ), demonstrating the introduction of F-containing chemicals. However, we did not find any peaks from new compositions in the XRD pattern (Fig. 3(c4)) because of the low sensitivity of X-ray diffraction meter system. Thus, FTIR and XPS were applied to detect the change of surface chemical compositions before and after PFOA modification. In the FTIR spectra shown in Fig. 4(a), new peaks attributed to the $-\mathrm{CF}$ stretching vibrations of $\mathrm{CF}_{2}$ and $-\mathrm{CF}_{3}$ groups were detected at the wave length of 1241,1138 and $1046 \mathrm{~cm}^{-1}$ on the PFOA-modified samples.[37, 41, 42]. Fig. 4(b) shows the XPS spectra of the sample after PFOA modification, the $F$ KL1 and F 1s peaks were observed at the binding energies of 833 and $692 \mathrm{eV}$, respectively. The high-resolution C 1s peaks ranging from 281 to $297 \mathrm{eV}$ are depicted in Fig.4 (c) and 4(d). The C 1s peaks of deposited layer were deconvoluted into three components at $288.7 \mathrm{eV}$,
$286.4 \mathrm{eV}$, and $284.8 \mathrm{eV}$, which are corresponding to $\mathrm{C}=\mathrm{O}, \mathrm{C}-\mathrm{O}$, and $\mathrm{C}-\mathrm{C}$ groups (Fig. 4(c)). In contrast, the $\mathrm{C} 1 \mathrm{~s}$ peaks after PFOAmodified surfaces could be resolved into five components: $-\mathrm{CF}_{3}$ at $293.8 \mathrm{eV},-\mathrm{CF}_{2}$ at $291.7 \mathrm{eV}, \mathrm{C}=\mathrm{O}$ at $288.8 \mathrm{eV}, \mathrm{C}-\mathrm{O}$ at $286.1 \mathrm{eV}$, and C-C at $284.8 \mathrm{eV}$ (Fig.4 (d)). Thus, both the FTIR and XPS measurements demonstrated that after PFOA modification, the Fcontaining groups, such as $-\mathrm{CF}_{2}$ and $-\mathrm{CF}_{3}$, were successfully introduced onto the deposited layer to reduce its surface energy significantly. Therefore, after PFOA modification, a minute amount of nickel perfluorooctanoate $\left(\mathrm{Ni}\left(\mathrm{CF}_{3}\left(\mathrm{CF}_{2}\right)_{6} \mathrm{COO}\right)_{2}\right)$ was generated and changed the porous textures on the origami ball into thin flake and flocculent structures. The corresponding chemical reaction equations were shown as follows:

$\mathrm{Ni}(\mathrm{OH})_{2}+2 \mathrm{CF}_{3}\left(\mathrm{CF}_{2}\right)_{6} \mathrm{COOH} \rightarrow \mathrm{Ni}\left(\mathrm{CF}_{3}\left(\mathrm{CF}_{2}\right)_{6} \mathrm{COO}\right)_{2}+2 \mathrm{H}_{2} \mathrm{O}$

$3 \mathrm{Ni}(\mathrm{OH})_{2} \cdot 2 \mathrm{H}_{2} \mathrm{O}+6 \mathrm{CF}_{3}\left(\mathrm{CF}_{2}\right)_{6} \mathrm{COOH} \rightarrow 3 \mathrm{Ni}\left(\mathrm{CF}_{3}\left(\mathrm{CF}_{2}\right)_{6} \mathrm{COO}\right)_{2}+8 \mathrm{H}_{2} \mathrm{O}$ 

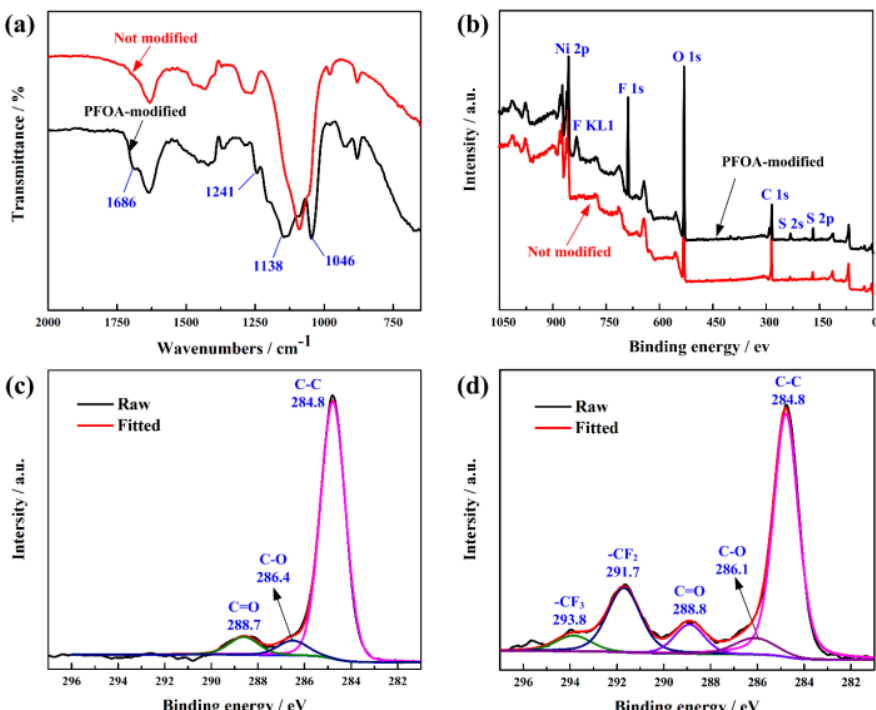

Fig. 4 (a) FTIR spectra and (b) XPS spectra of the chemically deposited microspheres before and after PFOA modification; (c)-(d) $C 1$ s peaks of the microspheres before and after PFOA modification.

To adjust and control the wettability of the stainless steel surfaces, the effect of chemical deposition time on oleophobicity were studied in detail. Fig. 5(a) to 5(d) respectively show the morphologies of the stainless steel surfaces deposited for $2 \mathrm{~min}, 5 \mathrm{~min}, 10 \mathrm{~min}$, and $30 \mathrm{~min}$. In comparison, origami-ball-like structures increased and formatted the hierarchical microsphere textures with the increase of the chemical
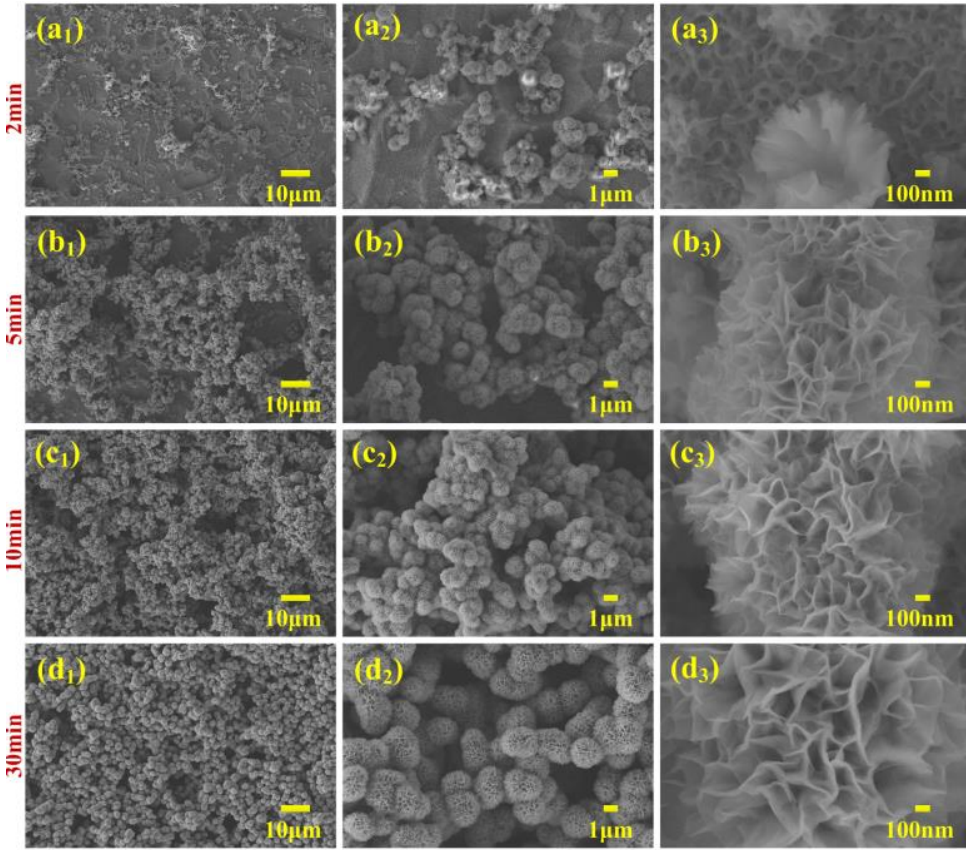

deposition time. In the view of the nanoscale structures, there were nearly no changes on the surface structures. At $2 \mathrm{~min}$ chemical deposition time, the CAs of water, glycerol, peanut oil and hexadecane were $158.0^{\circ}, 162.8^{\circ}, 147.3^{\circ}$ and $146.8^{\circ}$, respectively after the PFOA modification. The RAs of water, glycerol, peanut oil, and hexadecane were $9.45^{\circ}, 4.34^{\circ}, 12.92^{\circ}$, and $10.81^{\circ}$, respectively. It can be clearly seen that the samples showed super-repellence towards water and glycerol, but only high repellence for peanut oil and hexadecane which have lower surface tension. With a longer chemical deposition time, the shape of the four testing liquids on the samples gradually present as spheres indicating good superoleophobicity toward these liquids. In addition, the color of the deposited layer became black gradually because of the increased deposition substances with the prolonged deposition time. Fig. 5(e) and 5(f) shows the effect of deposition time on the CAs and RAs of the PFOAmodified chemically deposited surfaces. When the chemical deposition time was smaller than $5 \mathrm{~min}$, the CAs of water, glycerol, peanut oil, and hexadecane all showed an obvious increase with time and reached $160.3^{\circ}, 163.3^{\circ}, 158.7^{\circ}$ and $157.2^{\circ}$ at 5 min, respectively. Nevertheless, the RAs of the liquids saw a declined trend and got to $6.1^{\circ}, 4.0^{\circ}, 4.2^{\circ}$ and $5.4^{\circ}$ at $5 \mathrm{~min}$, respectively. During $5 \sim 10 \mathrm{~min}$ chemical deposition, the CAs and RAs were in a relatively stable level without obvious changes. Moreover, when the chemical deposition time prolonged beyond $10 \mathrm{~min}$, no changes in the CAs of water and glycerol appeared, but the CAs of peanut oil and hexadecane were slightly decreased. The RAs of water and glycerol show a small increase but they were still less than $10^{\circ}$, while significant increases appeared for the RAs of peanut oil and hexadecane. When the chemical deposition time was $30 \mathrm{~min}$, the RAs of peanut oil and hexadecane reached $41.3^{\circ}$ and $55.7^{\circ}$, respectively.

Fig. 5 (a)-(d) SEM images of the PFOA-modified stainless steel plates with different deposition times, the digital images of four types of liquids placed on these surfaces (the insets were the images of CAs); (e) to (f) changes in the CAs and RAs of the PFOA-modified chemically deposited surfaces as a function of chemical deposition time

In terms of the effect of the chemical deposition time on oleophobicity, an explanation was presented here. With the increase of the chemical deposition time, the number of the micro/nanometerscale origami-ball-like structures generated on the stainless steel substrates was also increased. Those structures formed re-entrant shapes and the air in the voids between origami-ball-like structures can support the oily liquids to achieve superoleophobicity, as shown in Fig. 6(a). With the increase of the chemical deposition time, the 
deposition substances increased from single layer to multiple layers, and changed from sparse to dense, as showed in Fig. 6(b). For hydrophobic materials, the adhesive force between the liquids and the surface increased with the real contact area.[43] In this study, to a certain extent, the real solid-liquid contact area increased with the chemical deposition time, leading to the rise of the adhesive force so that the RAs were increased. In the view of the difference between the surface tensions of water, glycerol, peanut oil and hexadecane, the RAs of water and glycerol with higher surface tensions increased only a little with chemical deposition time, whereas the RAs of peanut oil and hexadecane showed a big growth. (a)

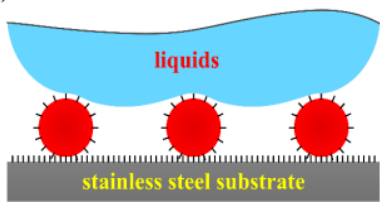

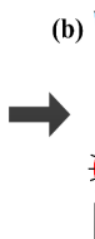

(b)

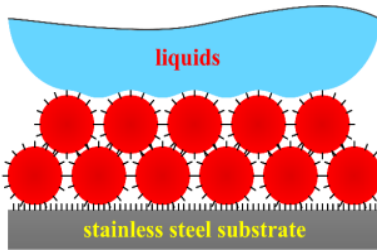

Fig. 6 Schematics of wetting behavior of the liquids on the sample surfaces with different deposition time: (a) 5-10 min; (b)>10 min.
3.2 High/low temperature storage and UV irradiation tests: To further demonstrate the ability of the obtained superoleophobic surfaces on stainless steel substrates to resist complex environment, high/low temperature storage and UV irradiation tests were carried out. Fig. 7(a) shows the water, glycerol, peanut oil and hexadecane CAs and RAs of the samples under different storage temperatures for $1 \mathrm{~h}$. The results clearly showed that the CAs of the four testing liquids all kept higher than $150^{\circ}$ and the RAs all lower than $10^{\circ}$ between a large temperature ranges, demonstrating that temperature had little effect on the superoleophobicity. The surfaces remained superoleophobic even after storing at low and high temperature environment (e.g. -30 and $100^{\circ} \mathrm{C}$ ), showing good thermal stability. Fig. 7(b) shows the water, glycerol, peanut oil and hexadecane CAs and RAs of the samples under UV exposure for different times. It was clearly shown that the CAs and RAs of the four testing liquids changed little during the UV irradiation. The reported steel surfaces retained superoleophobicity after exposure to high/low temperatures and UV irradiation, indicating that these surfaces have remarkable durability to harsh environment and great potential for practical applications.
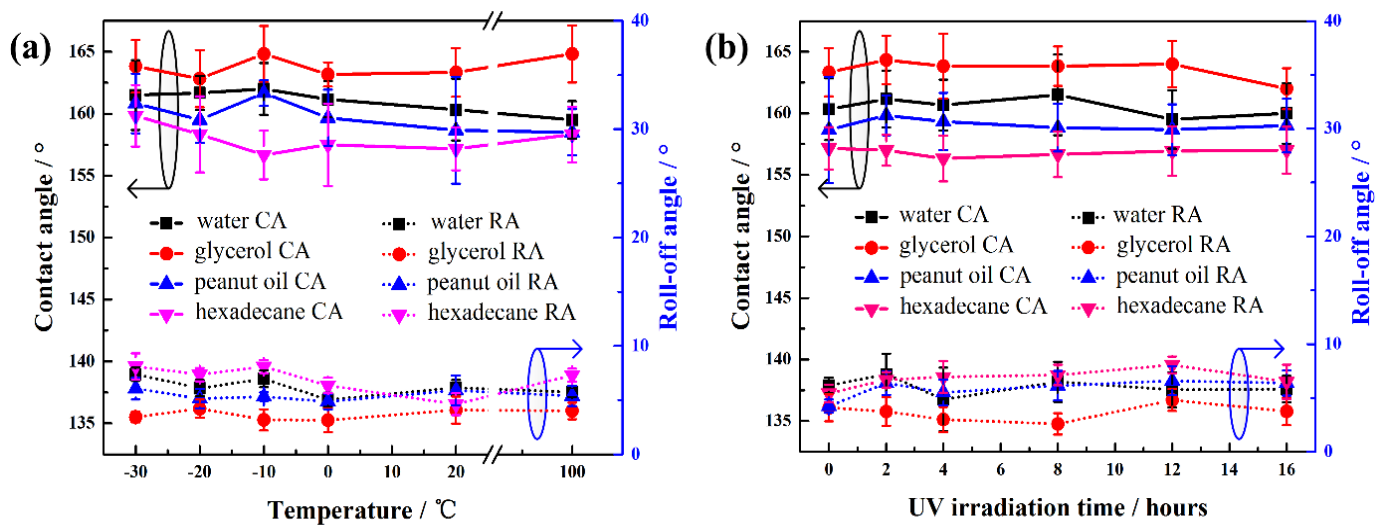

Fig. 7 Water, glycerol, peanut oil and hexadecane CAs and RAs of the samples in different circumstances: (a) different temperatures for 1 h; (b) UV irradiation $(365 \mathrm{~nm}, 32 \mathrm{~W})$

4. Conclusion: In summary, we developed a facile, efficient and low cost method to fabricate superoleophobic surfaces on stainless steel substrates that repel water, glycerol, peanut oil and hexadecane, using chemical bath deposition followed by low surface energy modification. The surface wettability could be simply controlled by chemical deposition time through adjusting the surface re-entrant morphologies. Through repeated experiments, $5 \mathrm{~min}$ of chemical deposition is the optimized time to achieve the highest CAs and lowest RAs of four liquids. The reported superoleophobic surfaces show remarkable stability when exposed to high and low temperatures, and UV irradiation and great potential to be practically applied in harsh environments.

5. Acknowledgements: This work was financially supported by National Natural Science Foundation of China (NSFC, Grant No.51605078 and No.51275071) and the Fundamental Research Funds for the Central Universities (DUT15RC(3)066).

\section{References:}

[1] Parkin I.P., Palgrave R.G.: 'Self-cleaning coatings', J. Mater. Chem., 2005, 15, (17), pp. 1689-1695.

[2] Crick C.R., Parkin I.P.: 'A single step route to superhydrophobic surfaces through aerosol assisted deposition of rough polymer surfaces: duplicating the lotus effect', J. Mater. Chem., 2009, 19,
(8), pp. 1074-1076.

[3] Zhao H., Law K.: 'Directional Self-Cleaning Superoleophobic Surface', Langmuir, 2012, 28, (32), pp. 11821-11827.

[4] Antonini C., Innocenti M., Horn T., Marengo M., Amirfazli A.: 'Understanding the effect of superhydrophobic coatings on energy reduction in anti-icing systems', Cold Reg. Sci. Technol., 2011, 67, (1-2), pp. 58-67.

[5] Boinovich L.B., Emelyanenko A.M., Ivanov V.K., Pashinin A.S.: 'Durable Icephobic Coating for Stainless Steel', ACS Appl. Mater. Interfaces, 2013, 5, (7), pp. 2549-2554.

[6] Guo P., Zheng Y., Wen M., Song C., Lin Y., Jiang L.: 'Icephobic/Anti-Icing Properties of Micro/Nanostructured Surfaces', Adv. Mater., 2012, 24, (19), pp. 2642-2648.

[7] Yao X., Song Y., Jiang L.: 'Applications of Bio-Inspired Special Wettable Surfaces', Adv. Mater., 2011, 23, (6), pp. 719-734.

[8] Kulinich S.A., Farzaneh M.: 'How Wetting Hysteresis Influences Ice Adhesion Strength on Superhydrophobic Surfaces', Langmuir, 2009, 25, (16), pp. 8854-8856.

[9] Farhadi S., Farzaneh M., Kulinich S.A.: 'Anti-icing performance of superhydrophobic surfaces', Appl. Surf. Sci., 2011, 257, (14), pp. 6264-6269.

[10] Lazauskas A., Guobiene A., Prosyevas I., Baltrusaitis V., Grigaliunas V., Narmontas P., Baltrusaitis J.: 'Water droplet behavior on superhydrophobic $\mathrm{SiO} 2$ nanocomposite films during 
icing/deicing cycles', Mater. Charact., 2013, 82, pp. 9-16.

[11] Wang B., Li J., Wang G., Liang W., Zhang Y., Shi L., Guo Z., Liu W.: 'Methodology for Robust Superhydrophobic Fabrics and Sponges from In Situ Growth of Transition Metal/Metal Oxide Nanocrystals with Thiol Modification and Their Applications in Oil/Water Separation', ACS Appl. Mater. Interfaces, 2013, 5, (5), pp. 1827-1839.

[12] Crick C.R., Gibbins J.A., Parkin I.P.: 'Superhydrophobic polymer-coated copper-mesh; membranes for highly efficient oilwater separation', J. Mater. Chem. A, 2013, 1, (19), pp. 59435948.

[13] Yang J., Zhang Z., Xu X., Zhu X., Men X., Zhou X.: 'Superhydrophilic-superoleophobic coatings', J. Mater. Chem., 2012, 22, (7), pp. 2834-2837.

[14] Changhong S., Youqian X., Wei Z., Yang L., Jun L.: 'Porous ceramic membrane with superhydrophobic and superoleophilic surface for reclaiming oil from oily water', Appl. Surf. Sci., 2012, 258, (7), pp. 2319-2323.

[15] Song J., Lu Y., Luo J., Huang S., Wang L., Xu W., Parkin I.P.: 'Barrel-Shaped Oil Skimmer Designed for Collection of Oil from Spills', Adv. Mater. Interfaces, 2015, 2, pp. 150035015.

[16] Nan Z., Kai L., Xiaohong L., Zhijun Z.: 'Preparation of superhydrophobic nano-silica aqueous dispersion and study of its application for water resistance reduction at low-permeability reservoir', Micro Nano Lett., 2012, 7, (6), pp. 526-528.

[17] Kashaninejad N., Nam-Trung N., Chan W.K.: 'Eccentricity effects of microhole arrays on drag reduction efficiency of microchannels with a hydrophobic wall', Phys. Fluids, 2012, 24, pp. 112004.

[18] Qian F.X., Jian N.W., Smith I.H., Sanderson K.D.: 'Directing the transportation of a water droplet on a patterned superhydrophobic surface', Appl. Phys. Lett., 2008, 93, (23), pp. 233112-233113.

[19] Mertaniemi H., Jokinen V., Sainiemi L., Franssila S., Marmur A., Ikkala O., Ras R.H.A.: 'Superhydrophobic Tracks for LowFriction, Guided Transport of Water Droplets', Adv. Mater., 2011, 23, (26), pp. 2911.

[20] Kang S.M., Lee C., Kim H.N., Lee B.J., Lee J.E., Kwak M.K., Suh K.: 'Directional Oil Sliding Surfaces with Hierarchical Anisotropic Groove Microstructures', Adv. Mater., 2013, 25, (40), pp. 5756.

[21] Feng L., Li S.H., Li Y.S., Li H.J., Zhang L.J., Zhai J., Song Y.L., Liu B.Q., Jiang L., Zhu D.B.: 'Super-hydrophobic surfaces: From natural to artificial', Adv. Mater., 2002, 14, (24), pp. 18571860.

[22] Ma M., Hill R.M.: 'Superhydrophobic surfaces', Curr. Opin. Colloid in, 2006, 11, (4), pp. 193-202.

[23] Kesong L., Milin Z., Jin Z., Jun W., Lei J.: 'Bioinspired construction of $\mathrm{Mg}-\mathrm{Li}$ alloys surfaces with stable superhydrophobicity and improved corrosion resistance', Appl. Phys. Lett., 2008, 92, (18), pp. 183101-183103.

[24] Xu W., Song J., Sun J., Lu Y., Yu Z.: 'Rapid Fabrication of Large-Area, Corrosion-Resistant Superhydrophobic Mg Alloy Surfaces', ACS Appl. Mater. Interfaces, 2011, 3, (11), pp. 44044414.

[25] Youfa Z., Jie W., Xinquan Y., Hao W.: 'Low-cost one-step fabrication of superhydrophobic surface on Al alloy', Appl. Surf. Sci., 2011, 257, (18), pp. 7928-7931.

[26] Li H.J., Wang X.B., Song Y.L., Liu Y.Q., Li Q.S., Jiang L., Zhu D.B.: 'Super-"amphiphobic" aligned carbon nanotube films', Angew. Chem. Int. Edit., 2001, 40, (9), pp. 1743-1746.

[27] Rahmawan Y., Xu L., Yang S.: 'Self-assembly of nanostructures towards transparent, superhydrophobic surfaces', J. Mater. Chem. A, 2013, 1, (9), pp. 2955-2969.

[28] Xu D., Mammen L., Butt H.J., Vollmer D.: 'Candle Soot as a
Template for a Transparent Robust Superamphiphobic Coating', Science, 2012, 335, (6064), pp. 67-70.

[29] Shuliang W., Changyu L., Guochao L., Ming Z., Jian L., Chengyu W.: 'Fabrication of superhydrophobic wood surface by a sol-gel process', Appl. Surf. Sci., 2011, 258, (2), pp. 806-810.

[30] Preda N., Enculescu M., Zgura I., Socol M., Matei E., Vasilache V., Enculescu I.: 'Superhydrophobic properties of cotton fabrics functionalized with $\mathrm{ZnO}$ by electroless deposition', Mater. Chem. Phys., 2013, 138, (1), pp. 253-261.

[31] Chengfeng J., Yufeng J., Tao N., Jianguo H.: 'Cellulose-based material with amphiphobicity to inhibit bacterial adhesion by surface modification', J. Mater. Chem., 2012, 22, (25), pp. 12562-12567.

[32] Tuteja A., Choi W., Ma M., Mabry J.M., Mazzella S.A., Rutledge G.C., McKinley G.H., Cohen R.E.: 'Designing superoleophobic surfaces', Science, 2007, 318, (5856), pp. 16181622.

[33] Choi W., Tuteja A., Chhatre S., Mabry J.M., Cohen R.E., McKinley G.H.: 'Fabrics with Tunable Oleophobicity', $A d v$. Mater., 2009, 21, (21), pp. 2190.

[34] Tuteja A., Choi W., McKinley G.H., Cohen R.E., Rubner M.F.: 'Design parameters for superhydrophobicity and superoleophobicity', Mrs Bull, 2008, 33, (8), pp. 752-758.

[35] Ahuja A., Taylor J.A., Lifton V., Sidorenko A.A., Salamon T.R., Lobaton E.J., Kolodner P., Krupenkin T.N.: 'Nanonails: A simple geometrical approach to electrically tunable superlyophobic surfaces', Langmuir, 2008, 24, (1), pp. 9-14.

[36] Song J., Huang S., Hu K., Lu Y., Liu X., Xu W.: 'Fabrication of superoleophobic surfaces on Al substrates', J. Mater. Chem. A, 2013, 1, (46), pp. 14783-14789.

[37] Chen F., Song J., Lu Y., Huang S., Liu X., Sun J., Carmalt C.J., Parkin I.P., Xu W.: 'Creating robust superamphiphobic coatings for both hard and soft materials', J. Mater. Chem. A, 2015, 3, (42), pp. 20999-21008.

[38] Zhiqing Y., Jiayu X., Chunqi W., Jingcheng Z., Suli X., Jun L.: 'Preparation of a superamphiphobic surface on a common cast iron substrate', J. Coating. Technol. Res., 2011, 8, (6), pp. 773777.

[39] Valipour Motlagh N., Birjandi F.C., Sargolzaei J., Shahtahmassebi N.: 'Durable, superhydrophobic, superoleophobic and corrosion resistant coating on the stainless steel surface using a scalable method', Appl. Surf. Sci., 2013, 283, pp. 636-647.

[40] Li J., Cheng H.M., Chan C.Y., Ng P.F., Chen L., Fei B., Xin A.J.H.: 'Superhydrophilic and underwater superoleophobic mesh coating for efficient oil - water separation', Rsc Adv., 2015, 5, (64), pp. 51537-51541.

[41] XiangHui X., ZhaoZhu Z., Weimin L.: 'Fabrication of superhydrophobic surfaces with perfluorooctanoic acid modified TiO 2/polystyrene nanocomposites coating', Colloids Surf. A, 2009, 341, (1-3), pp. 21-26.

[42] Li X., Zhang P., Jin L., Shao T., Li Z., Cao J.: 'Efficient Photocatalytic Decomposition of Perfluorooctanoic Acid by Indium Oxide and Its Mechanism', Environ. Sci. Technol., 2012, 46, (10), pp. 5528-5534.

[43] Yoon E.S., Singh R.A., Oh H.J., Kong H.: 'The effect of contact area on nano/micro-scale friction', Wear, 2005, 259, (SI2), pp. 1424-1431. 